\title{
Repression of BET activity sensitizes oral squamous cell carcinoma to PARP inhibition via inhibiting DNA homologous recombination and immune inhibition
}

\section{Dongdong Tong}

Shandong University

\section{Xin Zhang}

oral hospital of Shandong University

\section{Fenghe Zhang}

the oral hospital of Shandong University

xiaojing Liu (D 17865158237@163.com )

Shandong University https://orcid.org/0000-0002-1775-5747

\section{Primary research}

Keywords: Oral squamous cell carcinoma, PARP inhibitor, BET inhibitor, homologous recombination repair, immune escape

Posted Date: January 13th, 2021

DOI: https://doi.org/10.21203/rs.3.rs-26946/v2

License: (c) (i) This work is licensed under a Creative Commons Attribution 4.0 International License. Read Full License 


\section{Abstract}

Objective: To investigate the therapeutic resistance mechanism of oral squamous cell carcinoma (OSCC) to the PARP inhibitor olaparib and explore the combination with the BET inhibitor JQ1 to enhance its treatment effects.

Material and Methods: Cell proliferation, apoptosis and cell cycle distribution were evaluated. Levels of relevant factors were determined by quantitative real-time PCR, western blot and immunofluorescence. The effect of the combination treatment on xenograft OSCC mouse model was examined.

Results: Cal27 cells were more sensitive to the PARP inhibitor olaparib than Scc25 cells. Functional assays demonstrated that olaparib induced HR repair and upregulated PD-L1 expression, which results in drug resistance of OSCC to olaparib. Variations of these factors in the two cell lines may explain different sensitivity to olaparib. Moreover, the JQ1 BET inhibitor and olaparib synergistically exhibited anti-cancer effects in OSCC in vitro and in vivo and inhibited essential HR repair factors RAD51, BRCA1, and TOPBP1 through the ATR/CHK1 pathway and immune suppression mediated by the PD-L1 pathway.

Conclusions: Elevated HR and PD-L1 are involved in resistance mechanisms of OSCC to olaparib, attenuating its anti-tumor effects. Our results suggest that the strong synergistic anti-cancer effects of the combination of olaparib with the JQ1 BET inhibitor in OSCC in vitro and in vivo may be via suppression of the ATR/CHK1-mediated DNA damage response and PD-L1-related immune escape, indicating this combination strategy as a possible therapeutic approach for OSCC.

\section{Introduction}

OSCC is a usual cancer and affects nearly 500,000 patients annually worldwide [1]. Although recent advances in treatment methods of OSCC have generated promising avenues for therapeutic intervention, the average 5-year survival rate is still approximately $50 \%$ [2]. Therefore, more effective drugs are needed for OSCC patients.

The PARP enzyme family influences specific target proteins and plays an important role in wide range of cellular processes [3, 4]. Previous research demonstrated that PARP is correlated with the aggressiveness of many cancers including OSCC $[5,6]$, indicating that PARP inhibitors tend to be effective in cancer treatment. Although PARP inhibitors have shown promoting effects against some malignant tumors, research has shown that they remains a clinical hurdle due to the emergence of intrinsic and acquired resistance in some tumors [6-8]. Thus, preventing and overcoming PARPi resistance in patients would definitely improve the outcome of the treatment. Our study enclosed that PARP inhibitor olaparib was unable to inhibit proliferation at tolerated doses in OSCC cells and cell-derived xenografts when it was used as a single agent, underscoring the requirement for combination approaches. To date, however, the molecular mechanisms underlying tumor resistance to PARP inhibitors remain unclear. 
Homologous recombination (HR) is a kind of the most accurate DNA repair mechanisms which is not an all-or-nothing phenomenon, but that HR competency comes on a spectrum that ranges from complete deficiency to proficiency $[9,10]$. Previous studies showed that HR-deficient cancer cells are relatively more sensitive to the treatment of PARP inhibitors, which indicates that impairing the proficiency of HR in OSCC is crucial for maximizing treatment effects. RAD51, BRCA1 and TOPBP1 are three critical tumor suppressors crucial for DNA double strand break (DSB) repair and promoting the progression of cancers $[11,12][13,14]$. Therefore finding strategies which could selectively suppress these factors in tumors, sensitizing HR-proficient tumors to olaparib and benefiting the patients.

Current research has manifested that conventional anticancer therapies influence tumor-targeting immune responses and indicated that the treatment of PARP inhibitors are associated with immune escape, which has negative effects on drug responses in some tumors [15-17]. Thus, describing the crosstalk regarding anticancer treatments and drug-related immunity precisely could result in better combinatorial methods. Programmed death-1 (PD-1) and its ligand (PD-L1) take part in inhibiting autoimmunity, and their overexpression causes poor prognosis in many kinds of cancers [18-20].Although PD-1 and PD-L1 are particularly important in cancer progression, it is hard to regulate their expression as the use of immune checkpoint therapies targeting them continue to show limited durable success in clinical cases. Previous reports showed that there is an increase of PD1/PD-L1 as a reaction to DNA damage in some tumors [21, 22], which suggests that PD-L1 mediated pathway may also be regulated by DNA damage drugs including PARP inhibitors, but whether it plays a similar role in OSCC is still unknown.

BET family proteins can associate with mitotic chromosomes and control some significant genes through attracting transcription factors [23], BET bromodomain inhibitors including JQ1, exhibiting antitumor activities in a range of preclinical models of many tumors [24]. BRD4 have been implicated in DNA replication and DNA damage responses in some tumors and it was also augmented by PARPi in our study, which allowed us to hypothesize that, in OSCC, targeting BRD4 simultaneously could overcome PARPi resistance by depleting relative genes expressions.

In our experiments, we detected the resistance mechanisms of PARP inhibitor olaparib in OSCC, additionally, examining the potential benefit and valid evidence of combination treatment with the BET inhibitor such as JQ1.

\section{Materials And Methods}

\subsection{Cell culture}

OSCC cells were provided by Shanghai Ninth People's Hospital, culturing in high glucose DMEM (Hyclone, Logan, UT, USA) supplemented with $10 \%$ fetal bovine serum at $37^{\circ} \mathrm{C}$ in a humidified incubator containing $5 \% \mathrm{CO}_{2}$.

\subsection{Reagents and antibodies}


The BET inhibitor JQ1 and PARP inhibitor olaparib were bought from Selleck Chemicals (Houston, TX, USA).Relevant antibodies used in this study: primary antibodies against caspase 3 (\#9662), cleaved caspase 3 (\#9664), BRCA1 (\#9010), TOPBP1 (\#14342), P-CHK1 (\#2348), CHK1 (\#2360), ATR (\#13934), PD-L1 (\#13684), p53 (\#32389), p-p53 (\#211061) and secondary antibody (\#7076) from Cell Signal Technology (CST, Danvers, MA, USA); and anti-BRD4 (ab128874), anti-RAD51 (ab133534) and antiGAPDH antibodies (ab8226) from Abcam (St. Louis, MO, USA).

\subsection{Colony formation assay}

OSCC cells were cultured in 6-well plates at a density of 2000 cells/ well, and then treated Cal27 cells with $5 \mu \mathrm{M}$ olaparib $\pm 0.2 \mu \mathrm{M}$ JQ1, While dealing Scc25 cells with $20 \mu \mathrm{M}$ olaparib $\pm 1 \mu \mathrm{M}$ JQ1. After $48 \mathrm{~h}$ of culture, cells were fixed, with staining, photographing and counting subsequently.

\subsection{Cell viability assays}

Cell proliferation was detected with CCK-8 (Dojindo, Japan). Cells were cultured and treated with olaparib with or without JQ1 for 1-4 d. Next, adding CCK-8 solution and incubating for 0.5-1 h, after which measuring densities, at $450 \mathrm{~nm}$. The half maximal inhibitory concentration (IC50) was calculated by GraphPad Prism software (GraphPad Software, La Jolla, CA, USA) and combination index (CI) was acquired by CalcuSyn software.

\subsection{Apoptosis analysis}

OSCC cells were cultured at $5 \times 105$ cells/well and treated with olaparib, JQ1 or the combination for $2 \mathrm{~d}$. After staining with PE (BD Biosciences, San Jose, CA, USA), the proportion of apoptotic Cal27 and Scc25 cells were examined by FACS (BD Biosciences, Franklin Lakes, NJ, USA).

\subsection{Cell cycle analysis}

OSCC cells were seeded and treated by different inhibitors. After $48 \mathrm{~h}$, staining cells with propidium iodide (PI, BD Biosciences) and measuring cell cycle with a flow cytometer.

\subsection{Immunofluorescence}

OSCC cells were dealt with olaparib and JQ1 alone or in combination, and then cells were fixed with 4\% paraformaldehyde and $0.1 \%$ Triton X-100. Staining cells with rabbit anti-human RAD51 antibody (1:250) 
and PD-L1 antibody (1:250) overnight. After that cells were analyzed and images were obtained with a Leica fluorescence microscope.

\subsection{Flow cytometry}

Cal27 and Scc25 cells were cultured for $48 \mathrm{~h}$ in different inhibitors. Cells were trypsinized, washed with PBS solution, and blocked with PBS containing 10\% FBS. $2 \times 105$ cells were incubated with anti-PD-L1 and anti- RAD51 for $30 \mathrm{~min}$ at $4{ }^{\circ} \mathrm{C}$. After repeated washing, cells were resuspended in $500 \mu \mathrm{l}$ of PBS and analyzed on a FACS flow cytometer (BD Biosciences, Franklin Lakes, NJ, USA).

\subsection{Quantitative RT-PCR (qRT-PCR)}

OSCC cells were cultured and treated with olaparib, JQ1 or the combination for $48 \mathrm{~h}$. Extracting RNA with TRIzol reagent (TaKaRa Biotech, Shiga, Japan) and reversing transcribed into cDNA with Reverse Transcriptase kit (TaKaRa Biotech). qRT-PCR reactions were performed using the SYBR® Premix Ex Taq kit (TaKaRa Biotech) on a Roche 480 LightCycler (Basel, Switzerland). GAPDH mRNA served as the reference.

\subsection{Western blotting analysis}

After treating, cells were lysed in lysis buffer containing 1\% PMSF (Beyotime, Shanghai, China) for 30 min. Equal amounts of proteins were separated, with transferring to a $0.45 \mu \mathrm{m}$ membrane (GE HealthCare Life Science, Germany) for $1 \mathrm{~h}$ at $100 \mathrm{~V}$. Membranes were incubated in $3 \%$ nonfat milk for $1 \mathrm{~h}$ and then were incubated overnight at $4^{\circ} \mathrm{C}$ with primary antibodies. Membranes were then incubated with secondary antibodies for $1 \mathrm{~h}$. Immunoreactive bands were visualized by ECL detection reagents.

\subsection{Xenograft OSCC mouse models}

Six-week-old immunodeficient mice were purchased from Vital River Laboratories (Beijing, China). All mice were handled according to protocols approved by the ethics committee of Shandong University and Sichuan University. For experiments, $1 \times 107$ Cal27 and Scc25 cells were combined with Matrigel (Corning, NY, USA) injecting at the right flanks of mice. Mice were randomized into four groups and treated as follows when volume of tumors reached about 150-250 mm3, approximately one week later: control with vehicle (10\% captisol), JQ1 $(20 \mathrm{mg} / \mathrm{kg})$, olaparib $(50 \mathrm{mg} / \mathrm{kg})$ or the combination ( $\mathrm{n}=4$ per group). The gap time was two days between every injections and injected for 3 times in total. At the end of the experiments, dissecting tumors and analyzing. 


\subsection{Immunohistochemistry}

Tumor samples from mice were dissected and prepared according to the following protocol for immunohistochemical staining. Paraffin sections were deparaffinized in xylene, followed by 100, 95 and $70 \%$ ethanol. Antigen was unmasked by boiling in citric buffer. Endogenous peroxidase was removed from the sections by addition of $3 \% \mathrm{H} 2 \mathrm{O} 2$ in distilled water. Sections were blocked with monoclonal rabbit anti-human PD-L1 antibody (1:50; 13684T; Cell Signaling Technology) and RAD51 (1:200; Ab133534; ABCOM). After washing with PBS, slides were incubated with horseradish peroxidaseconjugated goat anti-rabbit IgG for $30 \mathrm{~min}$, followed by reaction with diaminobenzidine and counterstaining with Mayer's haematoxylin. On light microscopy, positive PD-L1 and RAD51 immunostaining was based on positive nucleus staining. The average integral optical density (IOD) of five randomly selected fields at $\times 400$ magnification was quantitatively assessed by using one-way ANOVA Immunostaining

\section{Results}

\subsection{Elevated HR and PD-L1 expression is responsible for PARPi resistance.}

According to our test in Fig3a by GraphPad Prism software, the IC50 values of olaparib in Cal27 and Scc 25 cells were $36 \mu \mathrm{M}$ and $98 \mu \mathrm{M}$ at 48h, respectively, indicating that the sensitivity of Cal27 cells to drug was relatively stronger than Scc25 cells. Then the CCK8 assays verified that olaparib alone had no significance effect on OSCC cells' survival. (Figure_1A)

To search for potential resistance mechanism, treating cells with olaparib at various concentrations and evaluated levels of RAD51, BRCA1 and TOPBP1, observing that the upregulation of these genes obviously compromised the olaparib-mediated killing effects. In Cal27 cells, $5 \mu \mathrm{M}$ olaparib treatment resulted in increased RAD51, BRCA1 and TOPBP1 (Figure_1B, 1C), causing drug resistance to olaparib as shown in Figure_1A. Similar results were observed in response to $10 \mu \mathrm{M}$, although to a lower degree compared with $5 \mu \mathrm{M}$ olaparib, which can help explain why the treatment effect of $10 \mu \mathrm{M}$ was better than $5 \mu \mathrm{M}$ olaparib in CCK8 assay. In Scc25 cells, olaparib at either $10 \mu \mathrm{M}$ or $20 \mu \mathrm{M}$ upregulated the expression of RAD51, BRCA1 and TOPBP1 obviously, so the treatment effect of olaparib was worse than that in Cal27 cells.

(Figure_1B)

To further explore the mechanism of olaparib resistance in OSCC, we examined the PD-L1 expression and it was consistent with HR-related proteins. (Figure_1B) We also examined the mRNA expressions of RAD51 and CD274 (PD-L1), which had the same trend of proteins in both cell lines. $(* * P<0.01, * P<0.05$, Figure_1C) 
Moreover, BRD4 expression was also upregulated by olaparib, which indicated the opportunity that we might use BET inhibitor to augment treatment effect of olaparib in OSCC. (Figure_1B)

Together these results indicated that the PARP inhibitor olaparib induced enriched HR-mediated DNA damage response as well as high PD-L1 expression, counteracting drug's killing effects and resulting in acquired resistance.

\subsection{BET inhibition impairs HR-mediated DNA damage repair and PD-L1-medicated immune escape}

To evaluate the HR efficiency and PD-L1 level in OSCC cells after treating by BETi, a dual experiments including Immunofluorescence and Western-blot were performed. The results showed that BRD4 pharmacological inhibition using JQ1 induced a dose-dependent reduction in RAD51, BRCA1 and TOPBP1 levels and was able to hinder homologous recombination-mediated DNA damage repair in OSCC cell lines, generating massive cell death and better treatment results. Moreover, the expression of PD-L1 was also prohibited by using JQ1 in a dose-dependent manner, which had similar reaction with HRrelevant factors, verifying that JQ1 could trigger immune inhibition in OSCC cells due to absence of BRD4. (Figure_2)

\subsection{BET inhibitor and PARP inhibitor synergistically suppress cell proliferation}

We next examined the effects of treating cells with olaparib, the BET inhibitor JQ1, or combining both inhibitors. The IC50 values of olaparib were calculated and they were robustly reduced after combination treatment ( $* * \mathrm{P}<0.01)$ (Figure_3A), indicating that OSCC cells became more sensitive to olaparib. $\mathrm{Cl}$ calculations confirmed that the combination JQ1 and olaparib treatment exhibited a synergistic effect (Table 1).

The JQ1 and olaparib treatment simultaneously also caused colony formation inhibition compared with other groups. (Figure_3B) The action of the combined treatment on proliferation was tested using CCK8 assays. The results showed that combination of two drugs cause a remarkable decline in cells' proliferation, comparing with individual inhibitors ( $* * P<0.01$, Figure_3C).

These results demonstrated that both olaparib and JQ1 at low concentrations had moderate impact on decreasing cell proliferation and viability of Cal27 and Scc25 cells when they were used alone, whereas simultaneously treated with JQ1 greatly triggered cell death and sensitized the OSCC cells to the drug. 


\subsection{BET inhibitor and PARP inhibitor synergistically induce apoptosis, which correlates with higher p53 levels}

We next examined whether the combination treatment affected the apoptosis of OSCC cells. Western blot analysis uncovered that the cleaved caspase- 3 which act as an important apoptosis marker was activated at a dramatically higher level in Cal27 and Scc25 cells after treating with JQ1 and olaparib. (**P $<0.01$, Figure_4A, 4B) Our research also evaluated cell apoptosis by Annexin V-PE staining followed by flow cytometry in OSCC cells treated with variable inhibitors for 2 days. Apoptotic cells accounted for higher proportions in response to the combination treatment than treatment by either inhibitor alone. ( ${ }^{\star} \mathrm{P}$ $<0.01$, Figure_4C, 4D)

P53 signaling is a key pathway that induces apoptosis. We thus next examined whether apoptosis was mediated by the p53 pathway. The combination treatment led to prominently increased p53 and phosphorylated p53 expressions compared with single treatments. $\left({ }^{*} \mathrm{P}<0.05,{ }^{\star \star} \mathrm{P}<0.01\right.$, Figure_4A, 4B)

Together our findings suggest that the combination of BET inhibitor and PARP inhibitor triggered a strong synergism in OSCC cells represented by a marked increase in cell apoptosis, which correlated with activation of the 553 pathway.

\subsection{BET inhibitor and PARP inhibitor co-treatment results in cell cycle arrest}

Then we focused on the effects of the combination treatment on cell cycle in OSCC cells. The results are displayed in Figure. 5A and quantitative analysis is shown in Figure. 5B. JQ1 and olaparib alone resulted in $\mathrm{G} 1$ or $\mathrm{G} 2 / \mathrm{M}$ phase cell cycle arrest, respectively, while the combination treatment caused $\mathrm{G} 1$ and $\mathrm{G} 2 / \mathrm{M}$ phase arrest as well as a significant decline in $\mathrm{S}$ phase cells.

Based on our data above, the simultaneous impairment of both HR and PD-L1 pathways is able to induce substantial cell cycle arrest in OSCC cells as shown by the co-treatment with JQ1 and ola.

\subsection{Combined JQ1/olaparib inhibition is effective in vivo}

Cal27 and Scc25 cells were injected into Six-week-old female mice and after the tumor volume reached approximately $150-250 \mathrm{~mm}^{3}$ after one week, mice were randomized into groups and treated with different inhibitors for 3 times. By the end of the 20 day treatment period, JQ1 and olaparib resulted in modest inhibition of tumor growth for both OSCC cell lines, and this was accompanied by minimal 
change in PD-L1 and RAD51 levels showed by immunohistochemical staining. The dual treatment was superior to any of the single treatments and resulted in significant tumor shrinkage, which also led to a marked reduction in the expression of RAD51 and PD-L1. (**P $<0.01$, Figure_6, 7) The differences were unlikely to be a result of generalized toxicity of drug treatment, since mouse weights were stable over the treatment period for all treatment groups.

The results demonstrated that combined treatment exhibits better antitumor effects in OSCC in vivo by inhibiting HR and PD-L1 pathway, compared with JQ1 or olaparib treatment alone.

\subsection{BET inhibitor and PARP inhibitor combination suppresses HR mediated by ATR/CHK1 pathway}

To explore the potential mechanism of the enhanced efficacy with combined treatment in OSCC, we examined several key DNA damage response factors involved in HR. Western blot showed that these HRrelated molecules were highly downregulated by the combination treatment compared with olaparib alone, although the expressions were slightly higher than in response to JQ1 alone. (Figure_8A) To better quantitate the cumulative expression of RAD51, we performed Flow cytometry and Immunofluorescence assays in Cal27 and Scc25 cells, respectively. Under fluorescence microscope and FACS flow cytometer, OSCC cells displayed the same level of the factor as seen in the Western blot. (Figure_8B, 8C) We also observed decreased RAD51 mRNA level in Cal27 and Scc25 cells treated with the combination therapy compared with olaparib monotherapy ( ${ }^{\star *} \mathrm{P}<0.01$, Figure_8D), which verified that JQ1 combined with olaparib can act by suppressing HR.

ATR/CHK1 plays a critical role in the regulation of replication and is essential in $\mathrm{HR}$, and thus we subsequently examined the ATR/CHK1 pathway. Consistent with our above results, the ATR/CHK1 pathway was increased in response to olaparib compared with controls, while the combination treatment reversely blocked this pathway compared with olaparib alone ( ${ }^{*} \mathrm{P}<0.01$, Figure_8A, $\left.8 \mathrm{D}\right)$, further indicating that JQ1 maximized olaparib's treatment effect through impairing HR mediated by ATR/CHK1 pathway inhibition.

These results indicate that the added BET inhibitor JQ1 disrupted HR via depleting ATR/CHK1 expressions, thus mitigating olaporib resistance and sensitizing the OSCC cell to the PARP inhibitor.

\subsection{BET inhibitor and PARP inhibitor combination attenuated expression levels of BRD4 and PD-L1}

We continued to explore the mechanism behind the combinatorial synergism of JQ1 and olaparib in OSCC cells. The use of JQ1 and olaparib synchronously reduced BRD4 expression to a greater extent compared with JQ1 monotherapy in Cal27 and Scc25 cells, which demonstrated that the sensitivity of 
OSCC to the BET inhibitor was also enhanced by the combination treatment. ( ${ }^{\star} P<0.05,{ }^{\star *} \mathrm{P}<0.01$ ) (Figure_9A, 9D)

We next determined whether BRD4 correlates with PD-L1 expression in OSCC by examining a panel of OSCC cell lines. We observed a trend towards a positive relationship between BRD4 and PD-L1 expression. (Figure_9A) We then examined whether BRD4 and CD274 expression was also positively correlated in OSCC cells. Indeed, there was a significant positive mRNA correlation between BRD 4 and CD274 in Cal27 and Scc25 cells treated with JQ1, olaparib or the combination. (Figure_9D) Notably, decreasing PD-L1 expression may be a strategy to optimize the antitumor effects of PARP inhibitors by adding BET inhibition. (Figure_9B, 9C)

Our results showed that the PARP inhibitor upregulated PD-L1 and BRD4 in OSCC cells, while BET blockade mitigated PD-L1-mediated immunosuppression activity, resulting in better treatment effect in OSCC cells. These results verified that the sensitivity of OSCC to the BET inhibitor was enhanced by the combination treatment with the PARP inhibitor.

\section{Discussion}

Combination of targeted therapies to augment treatment results is an effective therapeutic strategy for various cancers, including OSCC. PARP inhibitors have been the focus of intense investigation in cancer treatment, particularly as monotherapies or combining with conventional chemotherapeutics regarding DNA damage $[25,26]$, our results showed that the effects of PARP inhibitor olaparib in OSCC was moderate both in vitro and in vivo, which inspired us that identification of the mechanisms underlying resistance to olaparib and development of combination treatments that can produce potent responses in a preclinical setting of OSCC is required.

The DNA damage response exists in resistance mechanisms to anticancer treatment in many tumors. DNA damage response can be divided into two major pathways: the HR pathway for DSB repair and the nucleotide excision repair pathway for single-strand break repair [27, 28]. The ATR-CHK1 pathway is a central signal transduction processes that facilitates DNA repair and promotes cell proliferation. Activation of the ATR-CHK1 pathway can cause cell cycle arrest to allow cellular repair when the damage is irreparable [29] . In our study, the PARP inhibitor olaparib induced levels of several important HR-related factors in Cal27 and Scc25 cells, indicating that HR is induced in response to olaparib. We further found that olaparib upregulates the expression of the ATR/CHK1 pathway, which may contribute to HR repair in OSCC cells. Thus, we propose that olaparib activates the ATR/CHK1 pathway to induce HR in OSCC.

In recent years, accumulating evidence has highlighted the role of immunity in patient response to anticancer agents [30,31]. Our results demonstrated that the PARP inhibitor exhibited low levels of tumor inhibition effects on OSCC, which may be due to the induction of HR-mediated DNA damage response. However, the role of PARP inhibition in cancer-associated immunity has been largely unknown. Our findings showed that treatment with the PARP inhibitor olaparib alone upregulated PD-L1 expression in OSCC cells, which may contribute to the reduced efficacy of PARP inhibitor through inducing immune 
escape. Thus, strategies that can block the PD-L1 pathway to restore anti-tumor immunity may help potentiate the effects of PARP inhibitors in tumor suppression. We also found that Cal27 OSCC cells were more sensitive to olaparib than Scc25 cells, which may be partly due to different levels of HR repair and PD-L1 expressions, as olaparib induced higher activation of these factors in Scc25 cells.

A number of BET inhibitors have been developed and are entering clinical trials. After wide range of drug screening in preliminary analyses, selecting the JQ1 small molecule inhibitor of BRD4 because JQ1 treatment alone can decrease the DNA damage response and PD-L1 expression. Tumor cell viability fell down dramatically via the addition of JQ1 to olaparib, manifesting that JQ1 play a role in sensitizing Cal27 and Scc25 cells to the PARP inhibitor. We also verified that the synergistic effects of the combination treatment are partly attributable to the promotion of apoptosis mediated by p38 pathway. Notably, while the olaparib-induced G2/M arrest was reduced in Scc25 cells upon co-treatment with JQ1 treatment, the S phase population also decreased as a result of JQ1-induced G1 phase arrest. Taken together, our data suggested that OSCC are sensitive to the BET and PARP inhibitor synergistic treatment both in cells and in mouse model. Our research is the first study to demonstrate the combination effect of BET suppression with PARP inhibition in OSCC.

We continued to explore the mechanism underlying the synergistic treatment in OSCC. We found that JQ1 and olaparib exhibited anti-cancer activities in OSCC both in vitro and in vivo via inhibiting HR repair induced by the ATR/CHK1 pathway and immune escape mediated by PD-L1 pathway. Moreover, olaparib alone upregulated BRD4 expression, which may help clarify the possibility of synergism of BET and PARP inhibitors. Further studies of the molecular consequences of the combination treatment of BET and PARP inhibitors in OSCC will be necessary to develop effective combinations with optimal therapeutic potential.

\section{Conclusions}

Above all, our research verified that HR repair and upregulated PD-L1 expression in Cal27 and Scc25 cells resulted in adaptive cellular response to the PARP inhibitor olaparib. Adding BET inhibitor effectively attenuates the ATR/CHK1-mediated DNA damage response and PD-L1-related immune escape, increasing sensitivity of OSCC to PARP inhibitors in vitro and in vivo. Our results provide evidence for using BET inhibitors to enhance therapeutic efficacy of PARP inhibitors to HR-proficient and PD-L1proficient tumor cells. (Figure_10) The relationships between PARP inhibitors, HR and PD-L1 is timely and provides strong rational to explore more effective method consisting of PARP and BET inhibitors.

Cumulatively, this work provides valuable insights on the mechanisms of acquired resistance to olaparib and explores combinatorial therapeutic approaches involving bromodomain inhibitors rather than monotherapy, in order to augment the therapeutic strategies of PARP inhibitors. These results suggest a potential clinical benefit of the combination strategy for OSCC patients and may have far-reaching implications for OSCC patients in the future.

\section{Declaration}


Ethics approval and consent to participate

The work was finished according to protocols approved by the ethics committee of Shandong University

\section{Conflict of interest}

The authors have no potential conflicts of interest in the work.

\section{Consent for publication}

Not applicable

\section{Authors' contributions}

Xiaojing Liu participated in the conception and design of the study. Fenghe Zhang performed the data analysis and re-wrote the article. Xin Zhang contributed the cell culture and treatment for the study. Dongdong Tong finished the additional experiments. All authors read and approved the final manuscript.

\section{Funding}

This research was supported by natural science foundation of Shandong province (ZR2018MH023).

\section{Acknowledgments}

Not applicable

\section{Data Availability Statement}

The datasets used and analyzed during the current study are available from the corresponding author on reasonable request.

\section{Abbreviations}

OSCC Oral squamous cell carcinoma

HR Homologous recombination

DSB DNA double strand break

PD1 Programmed cell death-1

ATR Rad3-related kinase

CHKI Checkpoint kinase 1

BET Bromodomain and extra-terminal domain 


\section{References}

1. Csősz É, Lábiscsák P, Kalló G, Márkus B, Emri M, Szabó A, Tar I, Tőzsér J, Kiss C, Márton I:

Proteomics investigation of OSCC-specific salivary biomarkers in a Hungarian population highlights the importance of identification of population-tailored biomarkers. PLOS ONE 2017, 12(5).

2. Hu Q, Wu T, Chen X, Li H, Du Z, Hao Y, Peng J, Tai S, Song M, Cheng B: The poor outcome of second primary oral squamous cell carcinoma is attributed to Bmi1 upregulation. Cancer Medicine 2018, 7(4):1056-1069.

3. Navarro J, Gozalbo-López B, Méndez AC, Dantzer F, Schreiber V, Martínez C, Arana DM, Farrés J, Revilla-Nuin B, Bueno MF et al: PARP-1/PARP-2 double deficiency in mouse T cells results in faulty immune responses and T lymphomas. Scientific Reports 2017, 7.

4. Langelier MF, Riccio AA, Pascal JM: PARP-2 and PARP-3 are selectively activated by $\mathbf{5}^{\prime}$ phosphorylated DNA breaks through an allosteric regulatory mechanism shared with PARP-1. Nucleic Acids Research 2014, 42(12):7762-7775.

5. Curtin N, Szabo C: Therapeutic Applications of PARP Inhibitors: Anticancer Therapy and Beyond. Molecular aspects of medicine 2013, 34(6).

6. Berger NA, Besson VC, Boulares AH, Bürkle A, Chiarugi A, Clark RS, Curtin NJ, Cuzzocrea S, Dawson TM, Dawson VL et al: Opportunities for the repurposing of PARP inhibitors for the therapy of nononcological diseases. British Journal of Pharmacology 2018, 175(2):192-222.

7. Scott CL, Swisher EM, Kaufmann SH: Poly (ADP-Ribose) Polymerase Inhibitors: Recent Advances and Future Development. Journal of Clinical Oncology 2015, 33(12):1397-1406.

8. Ning J, Wakimoto H, Peters C, Martuza RL, Rabkin SD: Rad51 Degradation: Role in Oncolytic VirusPoly(ADP-Ribose) Polymerase Inhibitor Combination Therapy in Glioblastoma. JNCl Journal of the National Cancer Institute 2017, 109(3).

9. Sieverman KJ, Rine J: Impact of Homologous Recombination on Silent Chromatin in Saccharomyces cerevisiae. Genetics 2018, 208(3):1099-1113.

10. Krejci L, Altmannova V, Spirek M, Zhao X: Homologous recombination and its regulation. Nucleic Acids Research 2012, 40(13):5795-5818.

11. Jette N, Lees-Miller SP: The DNA-dependent protein kinase: a multifunctional protein kinase with roles in DNA double strand break repair and mitosis. Progress in biophysics and molecular biology 2015, 117(0):194-205.

12. Srivastava S, Dahal S, Naidu SJ, Anand D, Gopalakrishnan V, Kooloth Valappil R, Raghavan SC: DNA double-strand break repair in Penaeus monodon is predominantly dependent on homologous recombination. DNA Research: An International Journal for Rapid Publication of Reports on Genes and Genomes 2017, 24(2):117-128. 
13. Ogiwara H, Kohno T: CBP and p300 Histone Acetyltransferases Contribute to Homologous Recombination by Transcriptionally Activating the BRCA1 and RAD51 Genes. PLOS ONE 2012, 7(12).

14. Broering TJ, Alavattam KG, Sadreyev RI, Ichijima Y, Kato Y, Hasegawa K, Camerini-Otero RD, Lee JT, Andreassen PR, Namekawa SH: BRCA1 establishes DNA damage signaling and pericentric heterochromatin of the X chromosome in male meiosis. The Journal of Cell Biology 2014, 205(5):663-675.

15. Okuda A, Kurokawa S, Takehashi M, Maeda A, Fukuda K, Kubo Y, Nogusa H, Takatani-Nakase T, Okuda S, Ueda $\mathrm{K}$ et al: Poly(ADP-ribose) polymerase inhibitors activate the p53 signaling pathway in neural stem/progenitor cells. BMC Neuroscience 2017, 18.

16. Konecny GE, Kristeleit RS: PARP inhibitors for BRCA1/2-mutated and sporadic ovarian cancer: current practice and future directions. British Journal of Cancer 2016, 115(10):1157-1173.

17. Césaire $M$, Thariat J, Candéias SM, Stefan D, Saintigny $Y$, Chevalier F: Combining PARP Inhibition, Radiation, and Immunotherapy: A Possible Strategy to Improve the Treatment of Cancer? International Journal of Molecular Sciences 2018, 19(12).

18. Dong P, Xiong Y, Yue J, Hanley SJB, Watari H: Tumor-Intrinsic PD-L1 Signaling in Cancer Initiation, Development and Treatment: Beyond Immune Evasion. Frontiers in Oncology 2018, 8.

19. Sun WY, Lee YK, Koo JS: Expression of PD-L1 in triple-negative breast cancer based on different immunohistochemical antibodies. Journal of Translational Medicine 2016, 14.

20. Wang X, Wu WKK, Gao J, Li Z, Dong B, Lin X, Li Y, Li Y, Gong J, Qi C et al: Autophagy inhibition enhances PD-L1 expression in gastric cancer. Journal of Experimental \& Clinical Cancer Research : CR 2019, 38.

21. Sato H, Niimi A, Yasuhara T, Permata TBM, Hagiwara Y, Isono M, Nuryadi E, Sekine R, Oike T, Kakoti S et al: DNA double-strand break repair pathway regulates PD-L1 expression in cancer cells. Nature Communications 2017, 8.

22. Sun LL, Yang RY, Li CW, Chen MK, Shao B, Hsu JM, Chan LC, Yang Y, Hsu JL, Lai YJ et al: Inhibition of ATR downregulates PD-L1 and sensitizes tumor cells to T cell-mediated killing. American Journal of Cancer Research 2018, 8(7):1307-1316.

23. Taniguchi Y: The Bromodomain and Extra-Terminal Domain (BET) Family: Functional Anatomy of BET Paralogous Proteins. International Journal of Molecular Sciences 2016, 17(11).

24. Fu L, Tian M, Li X, Li J, Huang J, Ouyang L, Zhang Y, Liu B: Inhibition of BET bromodomains as a therapeutic strategy for cancer drug discovery. Oncotarget 2015, 6(8):5501-5516.

25. Gasche JA, Goel A: Epigenetic mechanisms in oral carcinogenesis. Future oncology (London, England) 2012, 8(11):1407-1425.

26. Smolensky D, Rathore K, Bourn J, Cekanova M: Inhibition of the PI3K/AKT Pathway Sensitizes Oral Squamous Cell Carcinoma Cells to Anthracycline-Based Chemotherapy In Vitro. Journal of cellular biochemistry 2017, 118(9):2615-2624.

27. Dabin J, Fortuny A, Polo SE: Epigenome maintenance in response to DNA damage. Molecular cell 2016, 62(5):712-727. 
28. Liu C, Vyas A, Kassab MA, Singh AK, Yu X: The role of poly ADP-ribosylation in the first wave of DNA damage response. Nucleic Acids Research 2017, 45(14):8129-8141.

29. Jung KW, Lee Y, Huh EY, Lee SC, Lim S, Bahn YS: Rad53- and Chk1-Dependent DNA Damage Response Pathways Cooperatively Promote Fungal Pathogenesis and Modulate Antifungal Drug Susceptibility. mBio 2019, 10(1).

30. Shi T, Ma Y, Yu L, Jiang J, Shen S, Hou Y, Wang T: Cancer Immunotherapy: A Focus on the Regulation of Immune Checkpoints. International Journal of Molecular Sciences 2018, 19(5).

31. Teige I, Mårtensson L, Frendéus BL: Targeting the Antibody Checkpoints to Enhance Cancer Immunotherapy-Focus on FcyRIIB. Frontiers in Immunology 2019, 10.

\section{Table}

Table_1 Synergistic induction of cell death by Olaparib and JQ1.

\begin{tabular}{|c|c|c|c|c|}
\hline \multirow{2}{*}{\multicolumn{2}{|c|}{$\mathrm{Cl}$ value(Cal27) }} & \multicolumn{3}{|c|}{ Ola( $\mu \mathrm{M})$} \\
\hline & & 5 & 10 & 20 \\
\hline \multirow{3}{*}{ JQ1 $(\mu M)$} & 0.2 & 0.03053 & 0.0441 & 0.03051 \\
\hline & 1 & 0.0427 & 0.02307 & 0.02582 \\
\hline & 2 & 0.21154 & 0.02109 & 0.02829 \\
\hline \multirow{2}{*}{\multicolumn{2}{|c|}{$\mathrm{Cl}$ value(Scc25) }} & \multicolumn{3}{|c|}{ Ola( $\mu \mathrm{M})$} \\
\hline & & 5 & 10 & 20 \\
\hline \multirow{3}{*}{ JQ1 $(\mu \mathrm{M})$} & 0.2 & 0.44457 & 0.6773 & 0.83529 \\
\hline & 1 & 0.34693 & 0.39597 & 0.27279 \\
\hline & 2 & 0.75134 & 0.53682 & 0.37592 \\
\hline
\end{tabular}

\section{Figures}




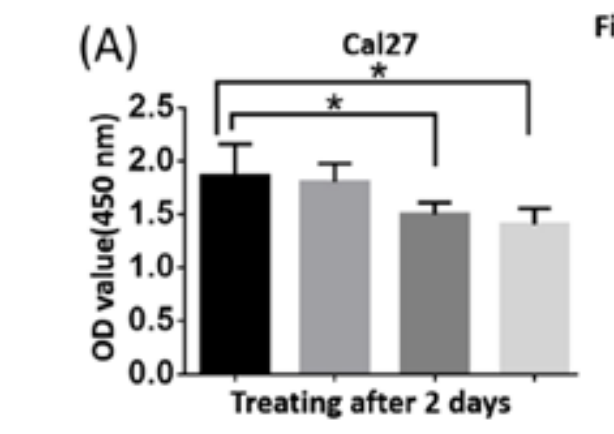

Fig. 1
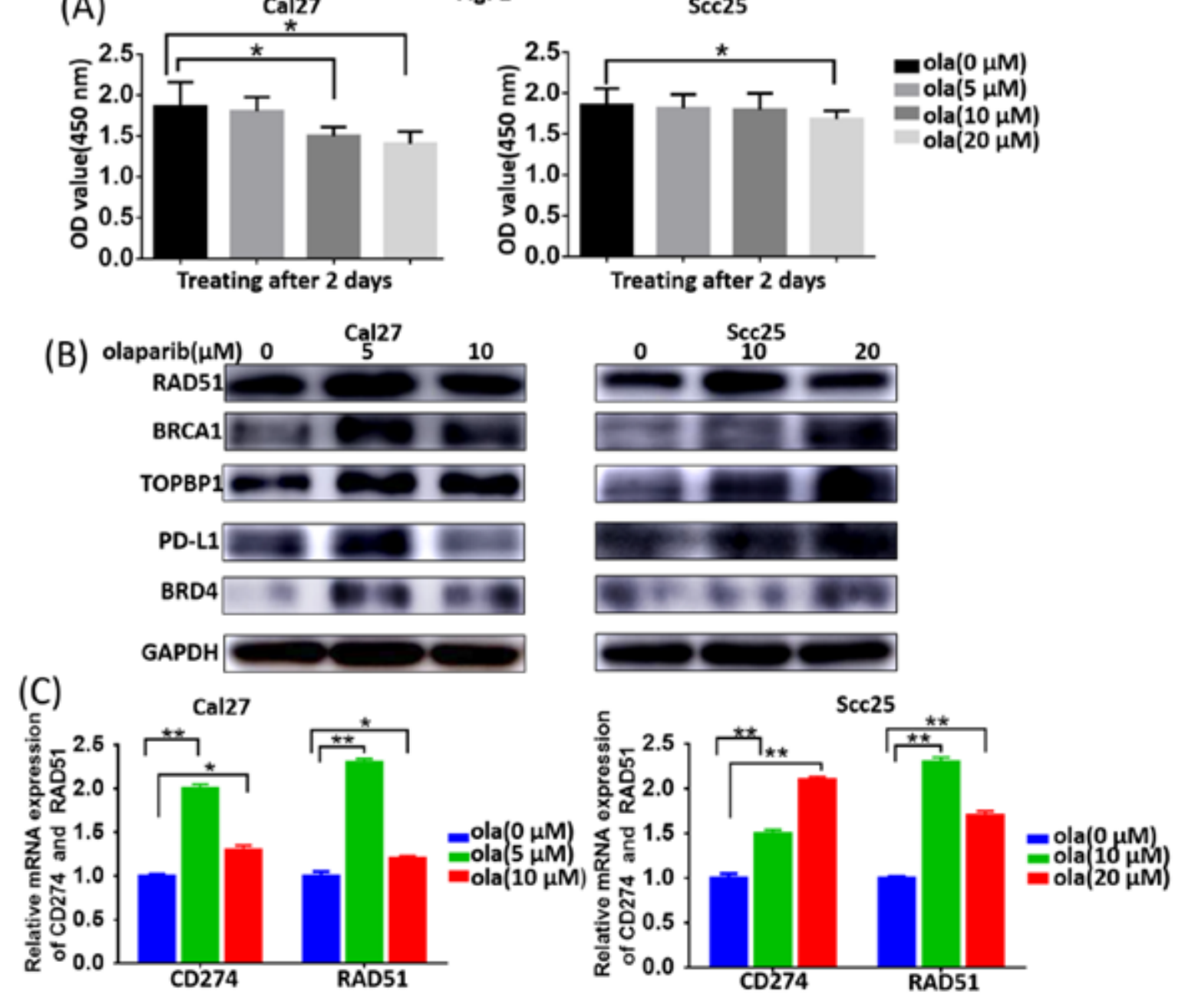

Figure 1

PARP inhibitor olaparib upregulates HR and PD-L1 in OSCC cells Cal27 and Scc25 cells were cultured for $24 \mathrm{~h}$ and then treated with olaparib at the concentrations of $0,5,10$, and $20 \mu \mathrm{M}$ for $2 \mathrm{~d}$. (A), CCK8 assay revealed that Cal27 cells are more sensitive to olaparib than Scc25 cells. (B), Relative protein expressions of RAD51, BRCA1, TOPBP1, PD-L1 and BRD4. GAPDH was used as a loading control. (C), Relative mRNA expressions of CD274 and RAD51 in Cal27 and Scc25 cells. Bars represent the mean \pm SD of each group $(n=6)$. Significant differences between groups are marked with asterisks. ${ }^{*}<0.05 * * P<0.01$ vs. control (the DMSO group). 


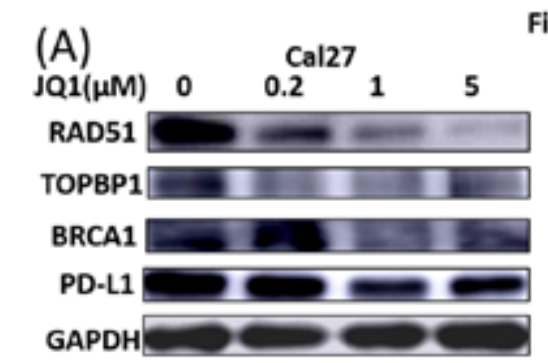

Fig. 2
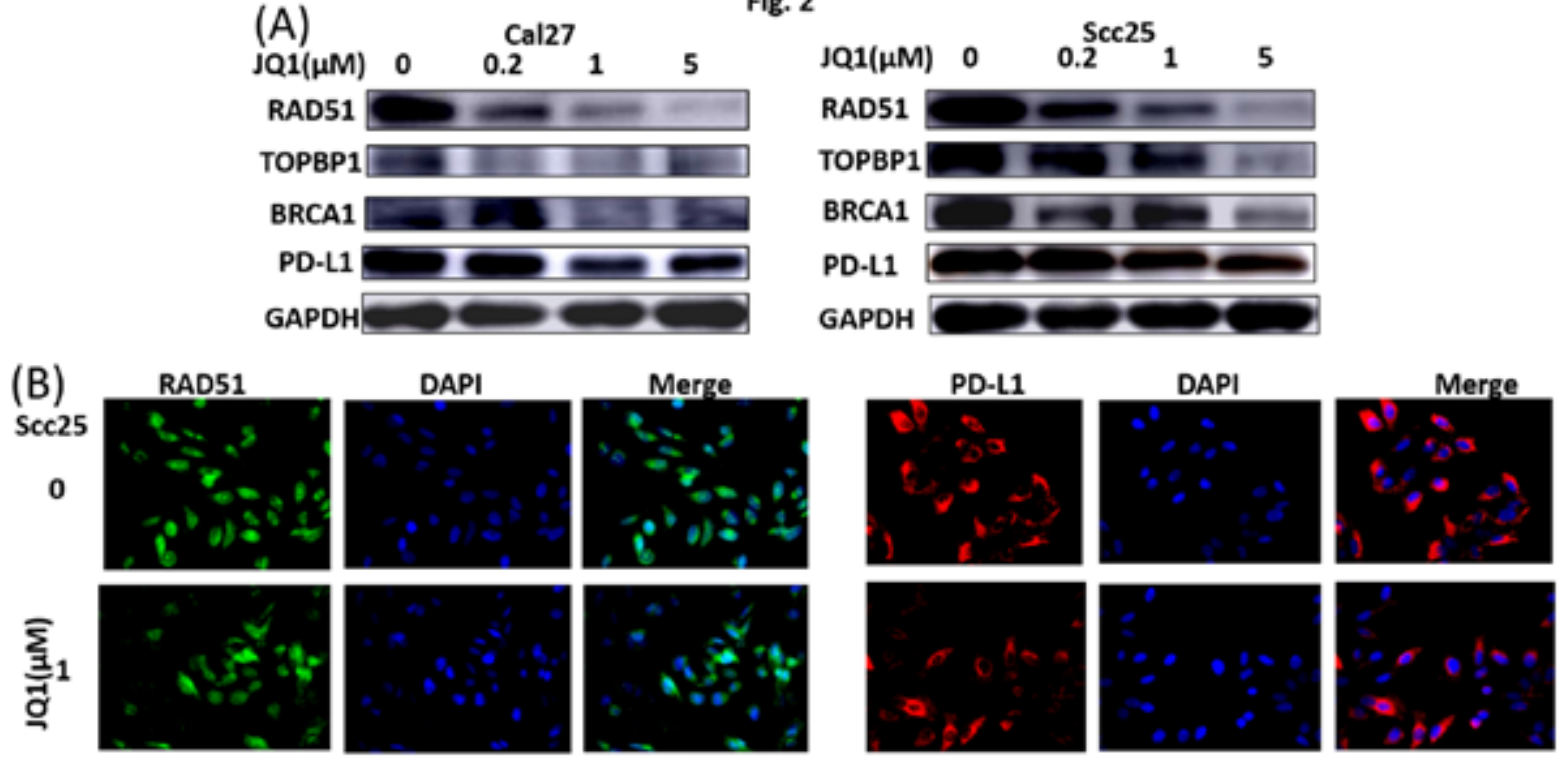

5
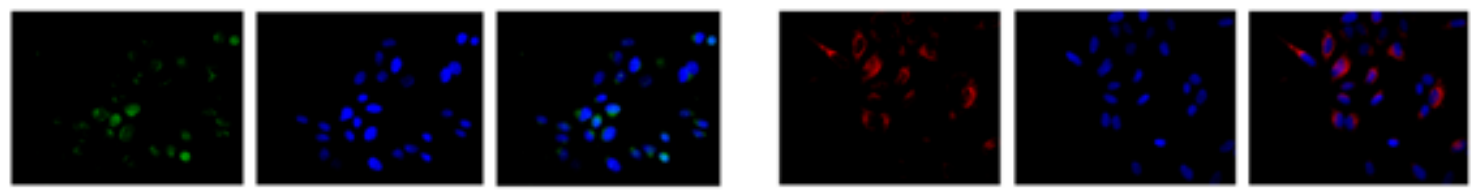

Figure 2

BET inhibition impairs DNA damage repair and PD-L1 expression Cal27 and Scc25 cells were cultured for $24 \mathrm{~h}$ and then treated with JQ1 at the concentrations of $0,0.2,1$, and $5 \mu \mathrm{M}$. (A), Relative protein expressions of RAD51, TOPBP1, BRCA1 and PD-L1. GAPDH was used as a loading control. (B), Immunofluorescent staining showed that JQ1 suppresses the expression of RAD51 in a dose-dependent manner. 


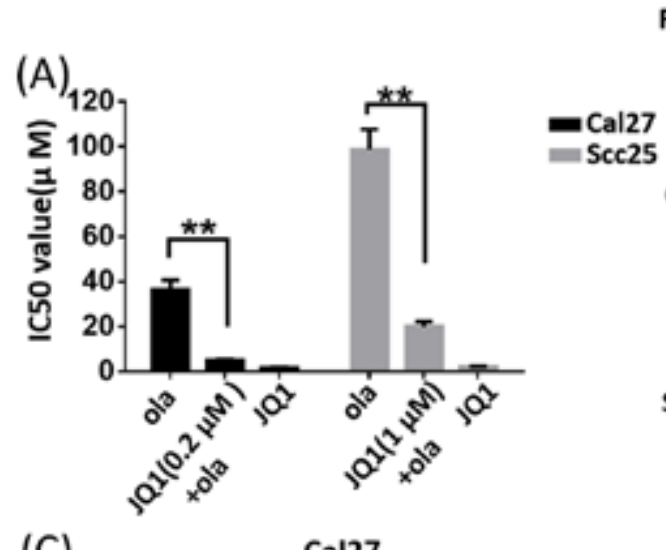

Fig. 3
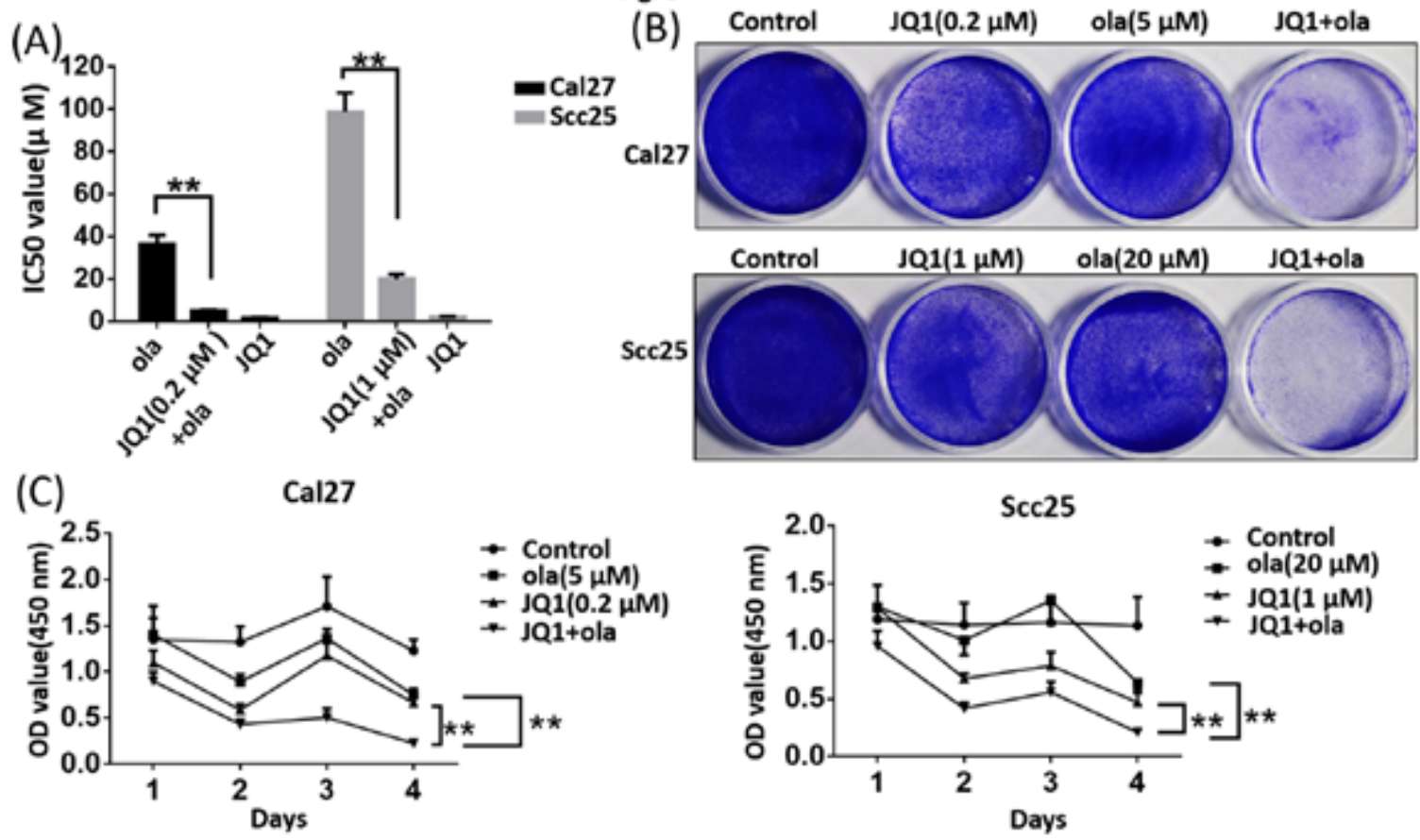

\section{Figure 3}

BET inhibitor and PARP inhibitor synergistically suppress cell proliferation Cal27 and SCC25 cells were cultured and then treated with JQ1, olaparib or the combination at different concentrations according to the $\mathrm{Cl}$ values. (A), The IC50 values of olaparib were significantly reduced after combination treatment in Cal27 and Scc25 cells. (B), Colony formation assay of Cal27 and SCC25 cell lines after treating for 2 days. (C), CCK8 assay revealed that combination treatment with JQ1 and olaparib inhibited Cal27 and SCC25 cell proliferation after treating for $1 \mathrm{~d}$ to $4 \mathrm{~d}$. Bars represent the mean \pm SD of each group $(n=6)$. Significant differences between groups are marked with asterisks. $(* \star P<0.01)$ 


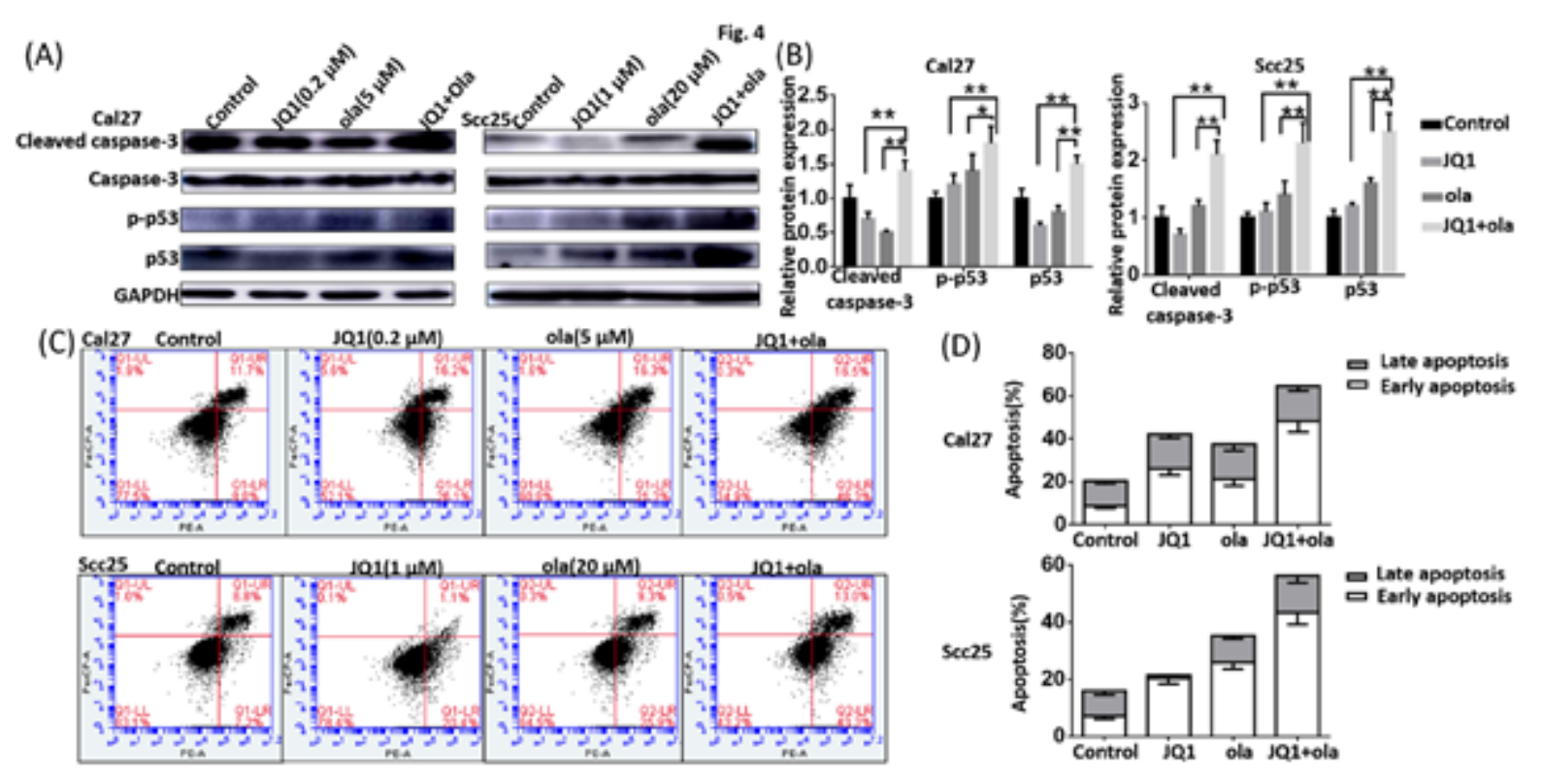

Figure 4

. BET inhibitor and PARP inhibitor synergistically induce apoptosis, which correlates with higher p53 levels Cal27 and SCC25 cells were cultured and then treated with JQ1, olaparib or the combination at different concentrations according to the $\mathrm{Cl}$ values for $2 \mathrm{~d}$. (A), Relative expressions of pro-apoptotic proteins including cleaved caspase-3, p53 and p-p53 upregulated while caspase-3 kept steady after combination treatment in Cal27 and Scc25 cells. GAPDH was used as a loading control. (B), Quantitative analysis of protein expressions of cleaved caspase-3, p-p53 and p53. (C), Cells then detected by flow cytometry assays using Annexin V-FITC/PE staining. Combination treatment by JQ1 and olaparib increased the apoptosis rate. (D), Sums of percentages of early apoptosis and late apoptosis were calculated as the total apoptosis ratios, which were graphically represented in the bar graphs. Bars represent the mean $\pm S D$ of each group $(n=6)$. Significant differences between groups are marked with asterisks. ( $\left.{ }^{\star} \mathrm{P}<0.05 * \star \mathrm{P}<0.01\right)$ 

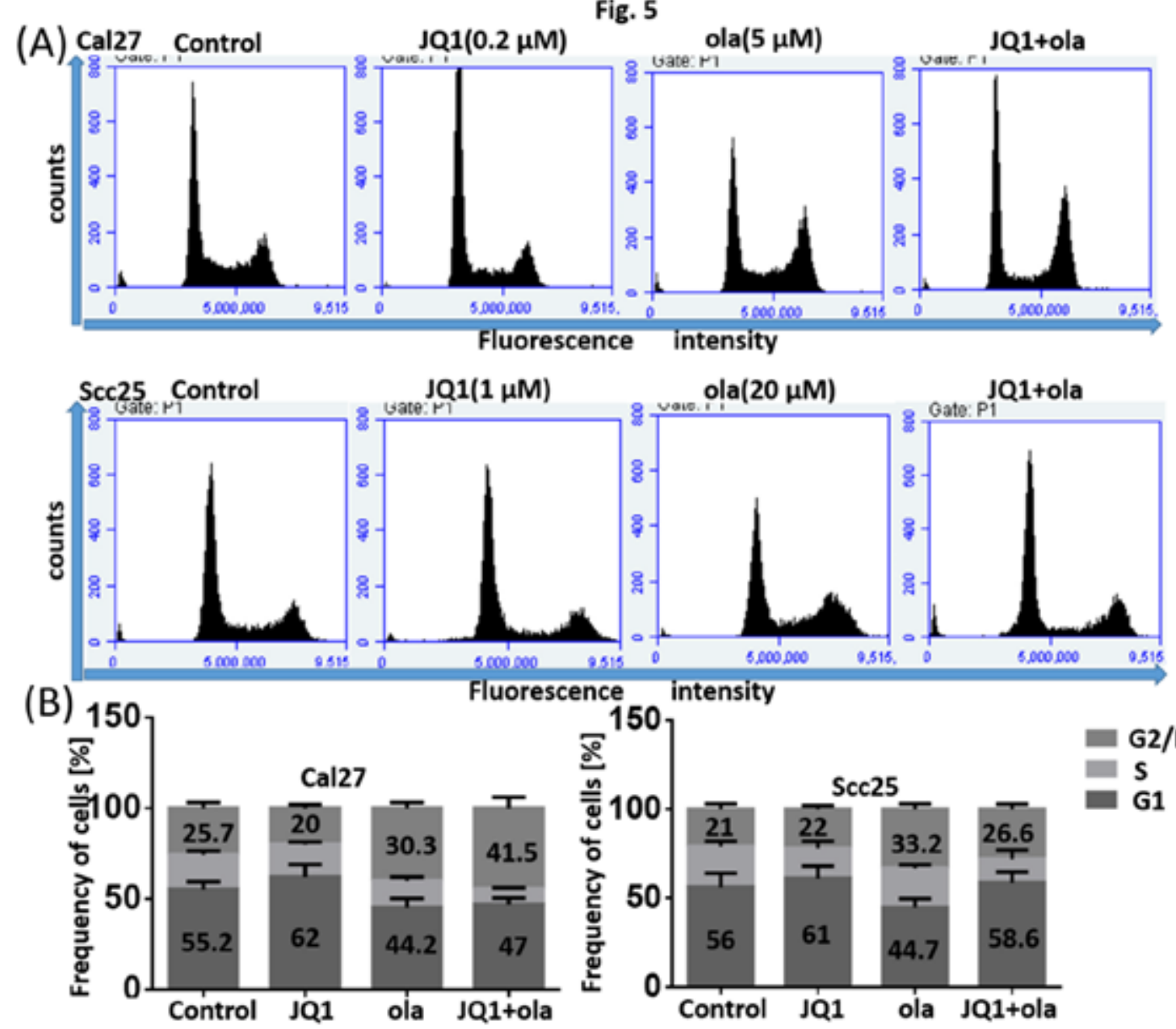

Figure 5

BET inhibitor and PARP inhibitor co-treatment causes cell cycle arrest Cal27 and SCC25 cells were cultured and then treated with JQ1, olaparib or $\mathrm{BET}$ inhibitor and PARP inhibitor co-treatment causes cell cycle arrest he combination for $2 \mathrm{~d}$. (A), Cells stained with PI and examined with flow cytometry assays to analyze cell cycle distribution. (B), Bar graphs showed the percentage of Cal27 and SCC25 cells in each cell cycle phase after treatment with JQ1 and/or olaparib. JQ1 and olaparib alone caused G1 and G2/M phase cell cycle arrest, respectively, while the combination treatment resulted in G1 and G2/M phase arrest and a profound reduction in S phase cells. Bars represent the mean \pm SD of each group $(n=6)$. 

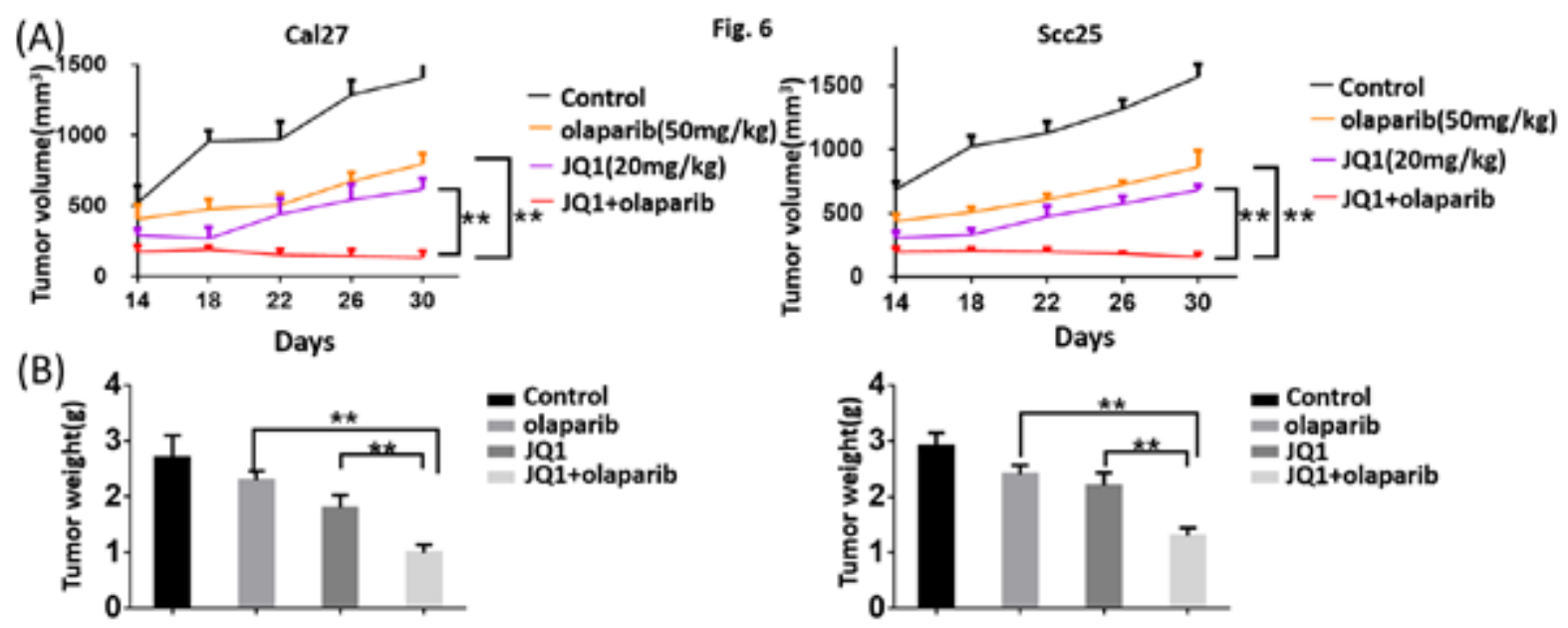

Figure 6

BET inhibitor and PARP inhibitor synergistically suppress the growth of tumors in vivo $1 \times 107$ Cal27 and Scc25 cells were mixed with equal volume of Matrigel and injected subcutaneously at the right flanks of mice, then mice were injected with JQ1, olaparib or the combination for 3 times. (A), Tumor growth curve of Cal27 xenograft in each group two weeks after exposure to JQ1, olaparib or the combination. (B), Weights of tumor from mice described above at sacrifice. $(\star \star P<0.01)$ 


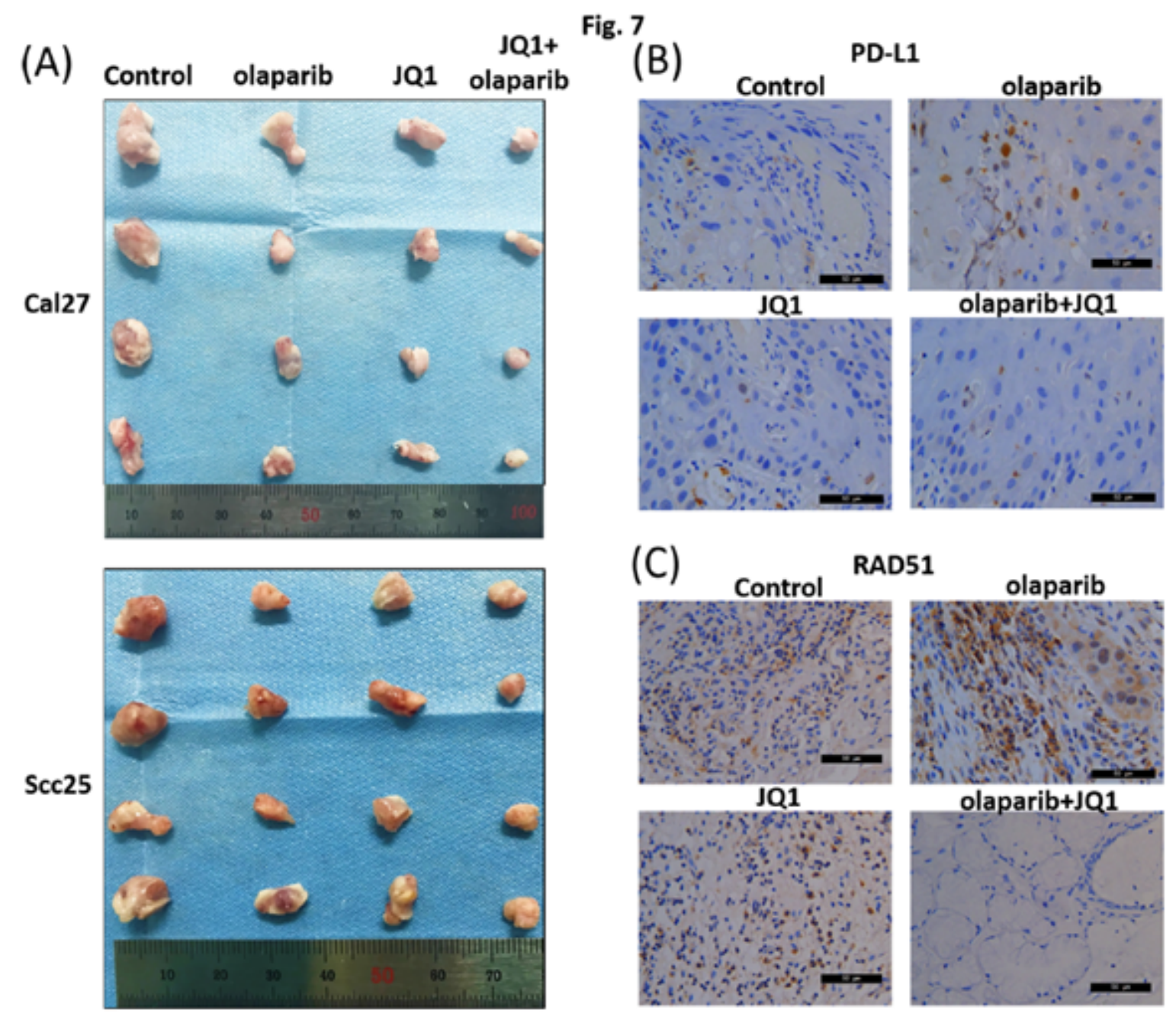

\section{Figure 7}

The effect of combination treatments in vivo is achieved by inhibiting PD-L1 and RAD51 (A), Image of representative tumors from Cal27 and Scc25 xenograft in each group. Bars represent the mean \pm SD of each group $(n=4)$. (B), Proteins were extracted from the tumors, then PD-L1 and RAD51 expression levels were analyzed by immunohistochemical staining. 


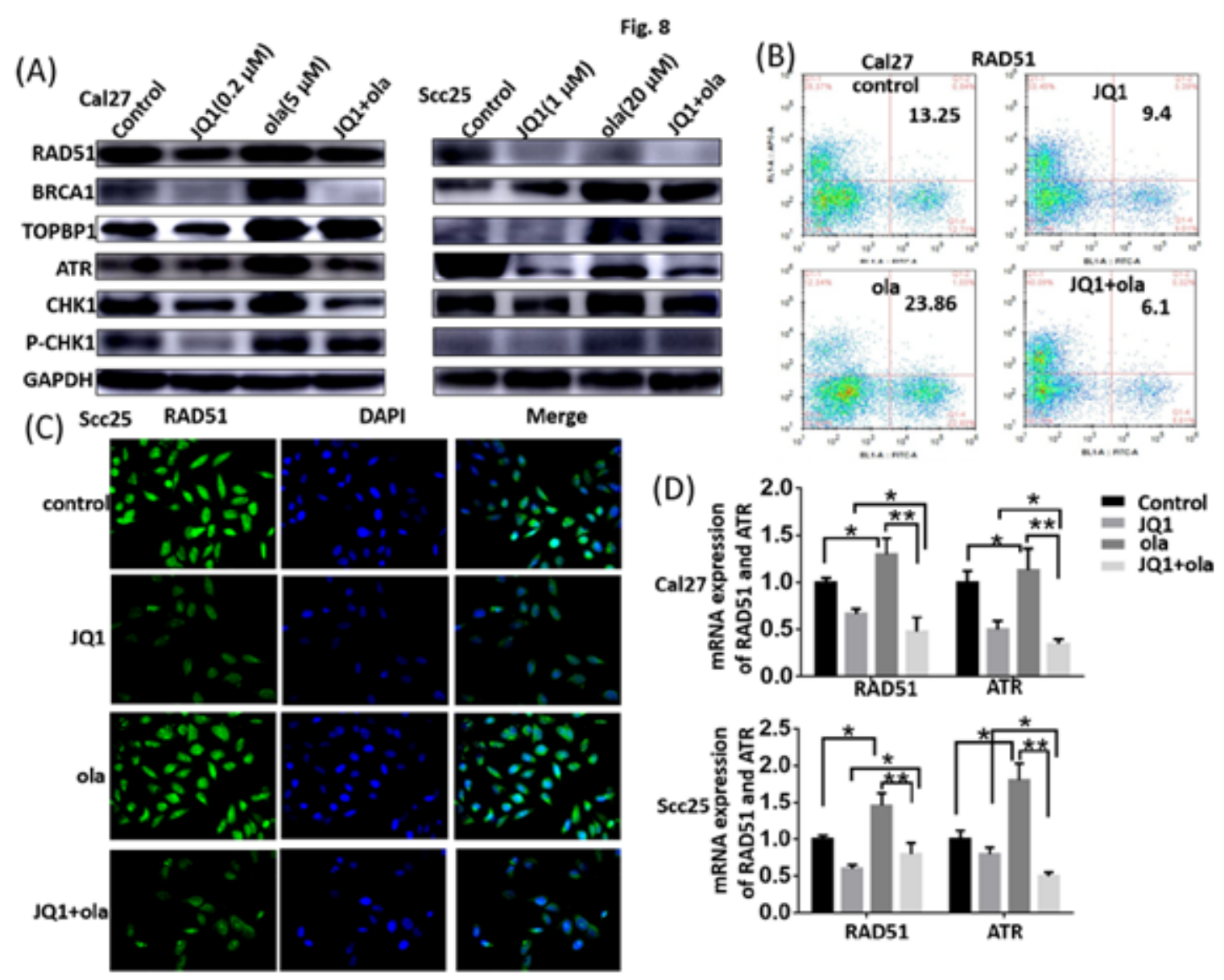

Figure 8

BET inhibitor and PARP inhibitor combination suppresses HR mediated by ATR/CHK1 pathway Cal27 and SCC25 cells were cultured and then treated with JQ1, olaparib or the combination for $2 \mathrm{~d}$. (A), Relative protein expressions of RAD51, BRCA1, TOPBP1, ATR, CHK1 and p-CHK1 in Cal27 and SCC25 cells. GAPDH was used as a loading control. (B), Under FACS flow cytometer, the expression of RAD51 was markedly inhibited after treating by JQ1 and Olaparib. (C), Immunofluorescent staining showed that combination treatment suppresses the expression of RAD51, compared with JQ1 or olaparib were used alone. (D), Relative mRNA expressions of RAD51 and ATR in Cal27 and Scc25 cells. Bars represent the mean $\pm S D$ of each group $(n=6)$. Significant differences between groups are marked with asterisks. $\left({ }^{*} \mathrm{P}<0.05\right.$ ** $\left.\mathrm{P}<0.01\right)$ 


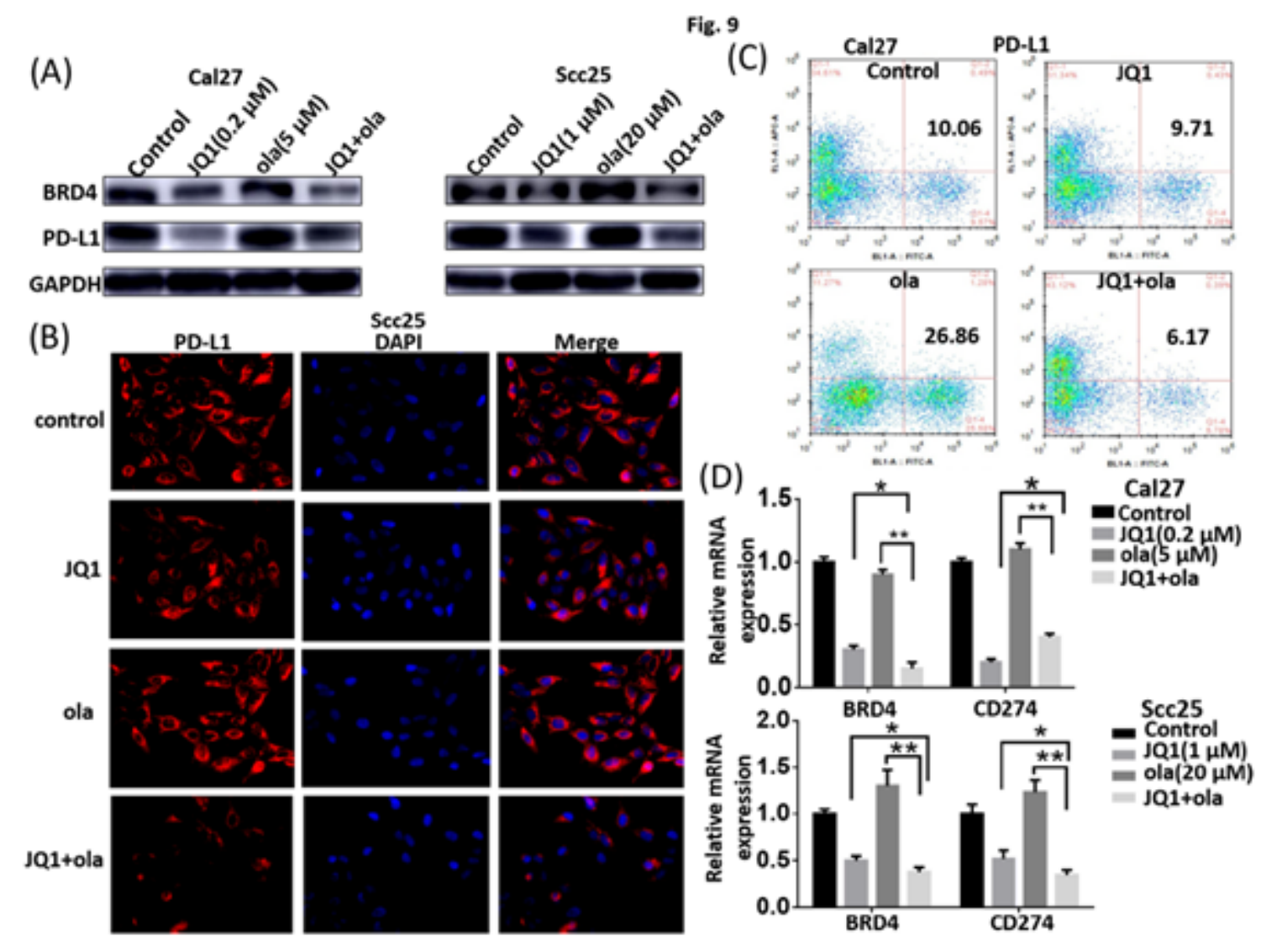

Figure 9

BET inhibitor and PARP inhibitor combination attenuated expression levels of BRD4 and PD-L1 Cal27 and SCC25 cells were cultured and then treated with JQ1, olaparib or the combination for $2 \mathrm{~d}$. (A), Relative protein expressions of BRD4 and PD-L1 in Cal27 and SCC25 cells. GAPDH was used as a loading control. (B), Immunofluorescent staining showed that combination treatment suppresses the expression of PD-L1, compared with JQ1 or olaparib were used alone. (C), Under FACS flow cytometer, the expression of PD-L1 was markedly inhibited after treating by JQ1 and Olaparib. (D), Relative mRNA expressions of BRD4 and CD274 after treating by different inhibitors. Bars represent the mean \pm SD of each group $(n=6)$.

Significant differences between groups are marked with asterisks. $\left({ }^{*} P<0.05 * \star P<0.01\right)$ 
Fig. 10

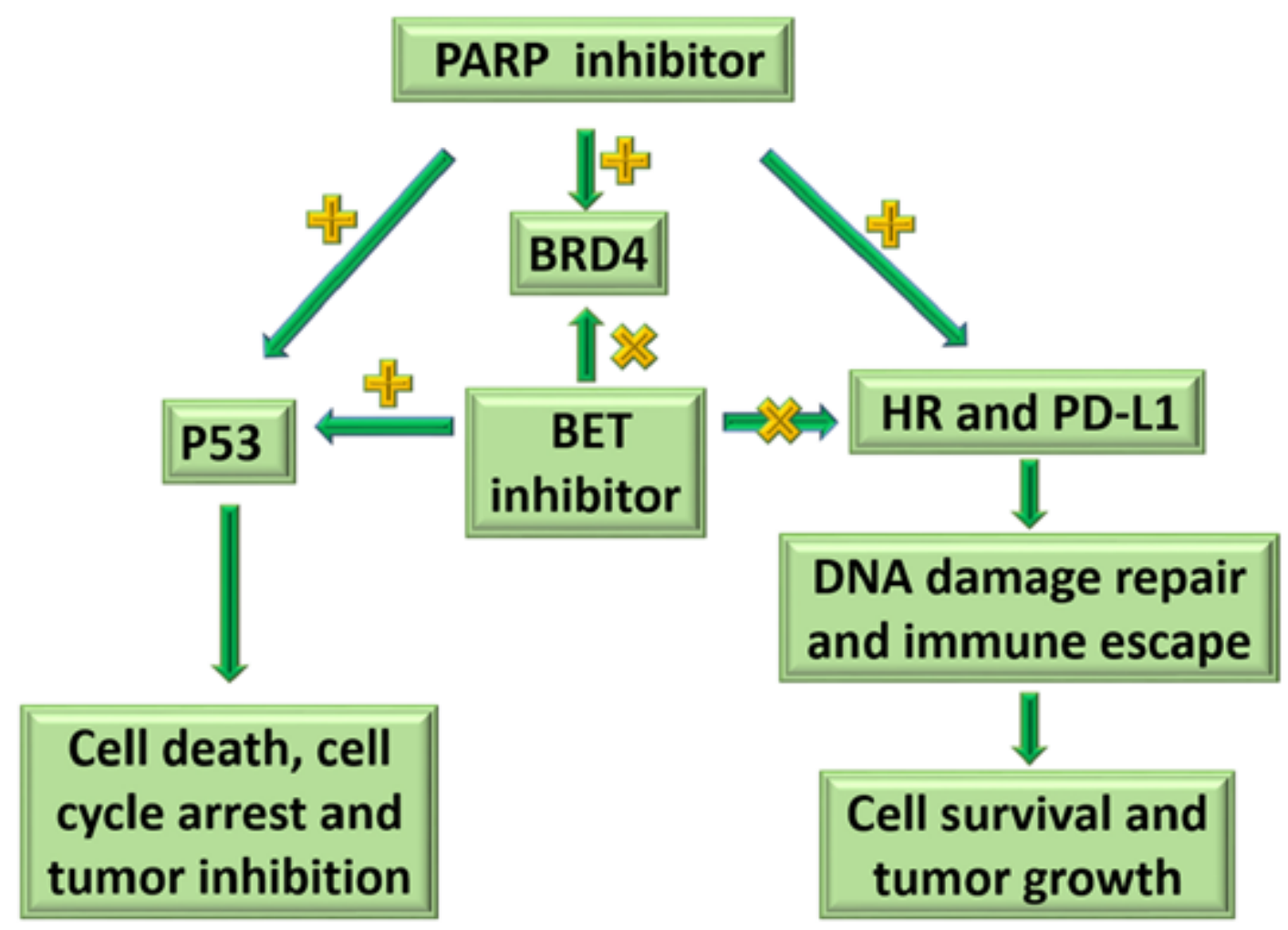

Figure 10

The mechanism figure of BET inhibitor augments treatment effects of PARP inhibitor in OSCC. Though PARP inhibitor has positive effect on p53-medicated cell death or cell cycle arrest, it also upregulates HRrelated DNA damage response and PD-L1-medicated immune escape which weaken treatment results in OSCC. Otherwise, BET inhibitor not only increases cell cycle arrest and induces cell death through p53 pathway, it also suppresses the expressions of HR and PD-L1, and so it provides scientific evidence to

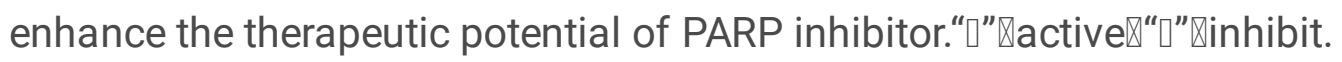

